\title{
A Manifesto for the Equifinality Thesis
}

\author{
Keith Beven \\ Lancaster University, UK
}

\begin{abstract}
This essay discusses some of the issues involved in the identification and predictions of hydrological models given some calibration data. The reasons for the incompleteness of traditional calibration methods are discussed. The argument is made that the potential for multiple acceptable models as representations of hydrological and other environmental systems (the equifinality thesis) should be given more serious consideration than hitherto. It proposes some techniques for an extended GLUE methodology to make it more rigorous and outlines some of the research issues still to be resolved.
\end{abstract}

Keywords: equifinality, GLUE, hydrological models, observation error, fuzzy measures, uncertainty

\section{Background}

In a series of papers from Beven (1993) on, I have made the case and examined the causes for an approach to hydrological modelling based on a concept of equifinality of models and parameter sets in providing acceptable fits to observational data. The Generalised Likelihood Uncertainty Estimation (GLUE) methodology of Beven and Binley (1992) which was developed out of the Hornberger-Spear-Young (HSY) method of sensitivity analysis (Whitehead and Young, 1979; Hornberger and Spear, 1981; Young, 1983), has provided a means of model evaluation and uncertainty estimation from this perspective (see Beven et al., 2000; Beven and Freer, 2001; Beven, 2001a for summaries of this approach). In part, the origins of this concept lie in purely empirical studies that have found many models giving good fits to data (e.g. Figure 1; for other recent examples in different areas of environmental modelling see Zak et al., 1999; Brazier at al., 2000; Beven and Freer, 2001a,b; Feyen et al., 2001; Mwakalila et al., 2001; Blazkova et al., 2002; Blazkova and Beven, 2002; Christiaens and Feyen, 2002; Freer et al., 2002; Martinez-Vilalta et al., 2002; Schulz and Beven, 2003). An independent example is provided by the results of Duan et al. (1992) from the University of Arizona group, although they have always rejected an approach based on equifinality in favour of finding better ways to find "optimal" models, most recently in a Pareto or Bayesian sense (e.g. Yapo et al., 1998; Gupta et al., 1998; Thiemann et al., 2001; Vrught et al. 2003). Despite this empirical evidence, however, many modellers are reluctant to adopt the idea of equifinality in hydrological modelling (and it can, indeed, always be avoided by concentrating on the search for an "optimum" but at the risk of avoiding important issues of model 
acceptability and uncertainty). This manifesto is an attempt to provide a convincing case as to why it should be embraced in future.

There is a very important issue of modelling philosophy involved that might explain some of the reluctance to accept the thesis. Science, including hydrological science, is supposed to be an attempt to work towards a single correct description of reality. It is not supposed to conclude that there must be multiple feasible descriptions of reality. The users of research also do not (yet) expect such a conclusion and might then interpret the resulting ambiguity of predictions as a failure (or at least an undermining) of the science. This issue has been addressed directly by Beven (2002a) who shows that equifinality of representations is not incompatible with a scientific research program, including formal hypothesis testing. In that paper, the modelling problem is presented as a mapping of the landscape into a space of feasible models (structures as well as parameter sets). The uncertainty does not lie in the predictions within this model space since the parameters in that space are known (even for a space of stochastic model structures). The dominant uncertainty lies in how to map the real system into that space of feasible models. Mapping to an "optimal" model is equivalent to mapping to a single point in the model space. Statistical evaluation of the covariance structure of parameters around that optimal model is equivalent to mapping to a small contiguous region of the model space. Mapping of Pareto optimal models is equivalent to mapping to a front or surface in the space of performance measures but which might be a complex manifold with breaks and discontinuities when mapped into in the model space. But, computer intensive studies of responses across the model space have shown that these mappings are too simplistic, since they arbitrarily exclude many models that are very nearly as good as the "optima". For any reasonably complex model, acceptably good fits are commonly found much more widely than just in the region of the "optimum" or Pareto "optima" (quotation marks are used here because the apparent global optimum may change significantly with changes in calibration data, errors in input data or performance measure).

This also brings attention to the problem of model evaluation and the representation of model error. The GLUE methodology has been commonly criticised from a statistical inference viewpoint for using subjective likelihood measures and not using a formal representation of model error (e.g. Clarke, 1994; Thiemann et al., 2001; and many different referees). For ideal cases, this can mean that non-minimum error variance (or non-maximum likelihood) solutions might be accepted as good models, that the resulting likelihoods do not provide the true probabilities of predicting an output given the model, while the parameter estimates might be biased by not taking the correct structural model of the errors into account in the likelihood measure. In fact, the GLUE methodology is general in that it can use "formally correct" likelihood measures if this seems appropriate (see Romanowicz et al., 1994; Romanowicz and Beven, 1996; and comments by Beven and Young, 2003), but need not require that any single model is correct (and correct here normally means not looking too closely at some of 
the assumptions made about the real errors in formulating the likelihood function, even if, in principle, those assumptions can be validated).

The difference is again one of philosophy. It is commonly forgotten that statistical inference methods were originally developed for fitting distributions to data in which the modelling errors can be treated as measurement errors, assuming that the chosen distributional form is correct. The approach is easily extended to regression and other more complex inference problems, but in each case, it is assumed that the model structure is correct and that the model errors can be treated as simple additive (or multiplicative if treated in log transform) measurement errors (see the difficulties that this can lead to in the discussion, for example, by Draper, 1995). Techniques such as reversible jump Monte Carlo Markov Chain methods have been developed to try to evaluate and combine the predictions of many potential model structures but in each case each individual model is treated as if it were correct. The "measurement error" terminology is still in use today in the calibration of complex simulation models (e.g. Thiemann et al., 2001 slip into this usage (p.2525) even though elsewhere they make clear the multiple sources of error), despite the fact that input data errors, model structural errors and other sources of error mean that the model errors are often much larger than sensible assumptions about measurement errors and despite the empirical evidence that there may not be a clear optimum in the model space.

So what are the implications of taking an alternative view, one in which it is accepted that the hydrological model (and the error model) may not be structurally correct and that there may not be a clear optimal model, even when multiple performance measures are considered? This situation is not rare in hydrological modelling. It is commonplace. It should, indeed, be expected because of the overparameterisation of hydrological models, particularly distributed models, relative to the information content of observational data available for calibration of parameter values (even in research catchments). But modellers rarely search for good models that are not "optimal". Nor do they often search for reduced dimensionality models that would provide equally good predictions but which might be more robustly estimated (e.g. Young, 2002). Nor do they often consider the case where the "optimal" model is not really acceptable (see, for example, Freer et al., 2002); it is, after all, the best available.

This paper tries to address some of these problems in the form of a manifesto for a future research programme. It starts with a brief summary of the causes of equifinality. It then considers the problem of parameter and uncertainty estimation in the ideal case of the perfect model. More realistic non-ideal cases are then discussed, together with techniques for model evaluation. The important issues of separation of uncertainties and model order reduction are identified as important for future research. 


\section{Equifinality, ambiguity, non-uniqueness, ill-posedness and identifiability}

The equifinality thesis is intended to focus attention on the fact that there are many acceptable representations that cannot be easily rejected and that should be considered in assessing the uncertainty associated with predictions. The concept owes a lot to the HSY analysis of multiple behavioural models in sensitivity analysis. The term equifinality has a long history in geomorphology, indicating that similar landforms might arise as a result of quite different sets of processes and histories. Thus from the landform alone, without additional evidence, it might be difficult to identify the particular set of causes or to differentiate different feasible causes (see discussion in Beven, 1996). The term was also used in the text of General Systems Theory of von Bertalanffy (1968) and was adopted for the environmental modelling context by Beven (1993). Implicit in this usage, is the rejection of the assumption that a single correct representation of the system can be found given the normal limitations of characterisation data.

For any particular set of observations, of course, some of those acceptable or behavioural models will be better in terms of one or more performance measures. The important point, however, is that given the sources of error in the modelling process, the behavioural models cannot easily be rejected as feasible representations of the system given the level of error in representing the system. In one sense, this can be viewed as a problem of decidability between feasible descriptions (hypotheses) of how the hydrological system is working (Beven, 2002a).

Decidability between models in hypothesis testing raises an interesting issue, however, linked to the information content of calibration data. To be able to represent different hypotheses about the processes of a hydrological system, it is necessary to have representations or parameterisations of those processes. This is why there has been a natural tendency for models to grow in complexity. Additional complexity will generally require additional numbers of parameters to be defined, the values of which will require calibration - but often without additional data being collected with the aim of determining those values (and in many cases, of course, direct measurement of parameters at the scale required by the model may not be possible, see Beven, 1989, 2002a,b). Thus, testing different hypotheses will tend to lead to more overparameterisation and equifinality and it should be expected that even if we could define the mathematically "perfect" model, it will still be subject to equifinality.

Environmental models are therefore mathematically ill-posed or ill-conditioned. The information content available to define a modelling problem does not allow a single mathematical solution. Non-uniqueness in model identification, particular for models that are complex relative to the quantity and quality of data available for model calibration, has also been used widely to indicate that multiple models might give equally acceptable fits to 
observational data. It has been primarily used in the discussion of the difficulties posed in parameter calibration for response surfaces that show many local minima, one of which may be (marginally) the global optimum, at least for that set of observations. Non-uniqueness (also non-identifiability) has usually been seen as a difficulty in finding the global optimal model and, by implication, the true representation of the system. It has not generally been viewed as an intrinsic characteristic of the modelling process.

Ambiguity has also been used to reflect model identification problems in a variety of ways . Beck and Halfon (1991) refer to ambiguity in distinguishing models identified on the same data set that have overlapping prediction limits. It is used somewhat differently by Zin (2002) to denote models for which predictions made with different stochastic realisations of the input data that cannot be distinguished statistically. Ambiguity is perhaps a less contentious word than equifinality but here the use of the latter is intended to emphasise that, given the normal limitations on the data for model evaluation, the decidability problem may be greater than statistical ambiguity between parameter sets but may also extend to real differences in process explanation when multiple model structures (or multiple functionality within a model structure) are considered.

Equifinality, ambiguity, non-uniqueness and ill-posedness have been discussed in this section with respect to the identifiability of models, parameter values and sets of parameter values. These terms are very often used in this way by modellers. It is, however, worth noting that there is another sense in which identifiability can be used in respect of environmental systems, i.e. whether the dominant modes of response of the system are identifiable. Hydrological systems, for example, often show relatively simple impulse response characteristics that can often be surprisingly well approximated by a linear transfer function (or unit hydrograph), even if the system gain may be nonstationary or nonlinear and difficult to predict (but see Young, 2001, 2003; Young et al., 2004). Where there is a dominant mode of response, it may well be possible to identify it relatively unambiguously. In one sense, the various parametric models that can be used to represent that response, with all their potential for equifinality and different process interpretations, are just different attempts to simulate the same response characteristics of the system. The ambiguity lies not in the system itself, but only in deciding about different representations of it (see for example the different explanations of "fractal" residence time distributions in Kirchner et al., 2001).

\section{Equifinality and the Deconstruction of Model Error}

That is not to say that any model error is arising totally from the different model representations of the system (model structures and parameter sets). There is a problem, in any modelling application, of trying to understand the origins of the error between model predictions of a variable and any observational data of the same variable. The difficulty 
comes because there are a variety of sources for the error but, at any given time, only one measure of the deviation or residual between prediction and observation at a site (i.e. the "model error"). Multiple observation sites or performance measures can, of course, produce conflicting prediction errors (an improvement in one prediction results in a deterioration in another). Thus, deconstruction of the error into its source components is difficult, particularly in cases common in hydrology where the model is nonlinear and different sources of error may interact in a nonlinear way to produce the measured deviation Beven, 2004b,c). There are obvious sources of error in the modelling process, for example, the error associated with the model inputs and boundary conditions, the error associated with using an approximate model of the real processes, and the error associated with the observed variable itself.

There are also some less obvious sources of error, such as the variable predicted by a model not being the same quantity as that measured, even though they might be referred to by the same name, because of heterogeneity and scale effects, nonlinearities or measurement technique problems (the incommensurability problem of Beven, 1989). A soil moisture variable, for example, might be predicted as an average over a model grid element several metres in spatial extent and over a certain time step; the same variable might be measured at a point in space and time by a small gravimetric sample, or by time domain reflectrometry integrating over a few tens of $\mathrm{cm}$, or by a cross-borehole radar or resistivity technique, integrating over several metres. Only the latter might be considered to approach the same variable as predicted by the model, but may itself be subject to a model inversion that involves additional parameters in deriving an estimate of soil moisture (see, for example, the discussion of instrument filters by Cushman, 1986, though this is not easily applied in nonlinear cases).

In rainfall-runoff modelling, the predictions are most usually compared with the measured discharges at the outlet from a catchment area. This may be considered to be the same variable as that predicted by the model, although it may be subject to measurement errors due to underflow or bypassing and rating curve inaccuracies, especially at very high and very low flows.

Since it is difficult to separate the sources of error that contribute to model error, as noted above it is often assumed to be adequately treated as a single lumped additive variable in the form:

$$
Q(X, t)=M(\Theta, X, t)+\varepsilon(X, t)
$$

where $Q(X, t)$ is a measured variable, such as discharge, at point $X$ and time $t ; M(\Theta, X, t)$ is the prediction of that variable from the model with parameter set $\Theta$; and $\varepsilon(X, t)$ is the model error at that point in space and time. Transformations of the variables of Eqn. (1) can also be used where this seems more appropriate to constrain the modelling problem to this form. 
Normal statistical inference then aims to identify the parameter set $\Theta$ that will be in some sense optimal, normally by minimising the residual error variance of a model of the model error, that might include its own parameters for bias and autocorrelation terms with the aim of making the residual error iid. This additive form allows the full range of statistical estimation techniques, including Bayesian updating, to be used in model calibration. The approach has been widely used in hydrological and water resources applications, including flood forecasting involving data assimilation (e.g. Krzysztofovicz, 2002; Young, 2002 and references therein); groundwater modelling, including Bayesian averaging of model structures (e.g. Neumann, 2003); and rainfall-runoff modelling (e.g. Kavetski et al., 2002; Vrugt et al., 2002, 2003).

In principle, the additive error assumption that underlies this form of uncertainty is particularly valuable for two reasons: that it allows checking of whether the actual errors conform to the assumptions made about the structural model of the errors and that, if this is so, then a true probability of predicting an observation, conditional on the model can be predicted. These advantages, however, may be difficult to justify in many real applications where poorly known input errors are processed through a nonlinear model subject to structural error and equifinality (see Hall, 2003, for a brief review of a more generalised mathematisation of uncertainty, including discussion of fuzzy set methods and the Dempster-Shafer theory of evidence). One implication of the limitations of the additive error model is that it may actually be quite difficult to estimate the true probability of predicting an observation, given one or more models, except in ideal cases.

\section{Ideal cases: theoretical estimation of uncertainty}

There are many studies in the hydrological literature, dating back to at least lbbitt and O'Donnell (1970) and continuing to at least Thiemann et al. (2001), where the effects of errors of different types on the identification of model parameters have been studied based on hypothetical simulation where it is known that the model is correct. This is the ideal case. A model run is made, given a known input series and known parameters, to produce a noise free set of "observations". The input data and observations are then corrupted by different assumed error models, generally with simple Gaussian structure, and a parameter identification technique is used to calibrate the model to see whether the original parameters can be recovered in the face of different types and levels of corruption. Any concerns about the level of model structural error can be neglected in such cases. The argument is that any model identification procedure should be shown to work for error corrupted ideal cases so that the user can have more faith in such a procedure in actual applications. This argument depends, however, on the application in practice not being distorted by model structural error (see next section). 
If the errors are indeed Gaussian in nature, or can be transformed to be, then the full power of statistical likelihood theory can be used. The simplest assumption, for the simulation of a single variable over a number of time steps $(T)$ is that the errors $\varepsilon(t)$ are an independent and identically distributed Gaussian variable with zero mean and constant variance. Then, the probability of predicting a value of $Q(t)$ given the model $M(\Theta)$ based on the additive model of Eqn.(1) is given by:

$$
\mathrm{L}(\mathrm{Q} \mid \mathrm{M}(\Theta)) \propto\left(\sigma_{\mathrm{e}}{ }^{2}\right)^{-\mathrm{T} / 2} \exp \left(\sigma_{\mathrm{e}}{ }^{2} / \sigma_{\mathrm{o}}{ }^{2}\right)
$$

where $\sigma_{\mathrm{e}}{ }^{2}$ is the variance of the error series, $\sigma_{\mathrm{o}}{ }^{2}$ is the variance of the observations, and T is the number of time steps.

The variance of the parameter estimates based on this likelihood function can be obtained from evaluating the Hessian of the log likelihood function at the point where the variance of the error series is minimised (or more generally where the log likelihood is maximised). Note, however, that for nonlinear models this will not produce the same result as evaluating (2) for every combination of parameters and using the estimate of the local error variance, even in the immediate region of the optimum. In this case a more direct evaluation of the nature of the likelihood surface using Monte Carlo, or Monte Carlo Markov Chain $\left(\mathrm{MC}^{2}\right)$ sampling techniques would be advantageous (e.g Kuczera and Parent, 1998, Vrugt et al. 2002)

For this ideal case, if the model fits the data very well and the error variance is very small, the likelihood function will be very peaked. This will be especially so if the model fits well over a large number of time steps (note the power of $T / 2$ in Eqn.(2)). The resulting variance of the parameter estimates will be very small. This arises out of the theory, regardless of whether there are other model parameter sets that produce error variances that are very nearly as small elsewhere in the model space.

This is a consequence of the implicit assumption that the optimal model is correct. In hypothetical ideal cases this is clearly so; but it is not such a good assumption in hydrological modelling of real catchment, groundwater or water quality systems. Simple assumptions about the error structures are convenient in applying statistical theory but are not often borne out by actual series of model errors which may show changing bias, changing variance (heteroscedasticity) and changing correlation structures under different hydrological conditions (and for different model structures or parameter sets). It is known for linear systems that ignoring such characteristics, or wrongly specifying the structure of the error model, will lead to bias in the estimates of parameter values. The same will be the case for nonlinear systems, but there is then no guarantee that, for example, Gaussian errors in model inputs will lead to an additive Gaussian error model of the form of Eqn.(1). 
There are ways of dealing with complex error structures within statistical likelihood theory; one is to try and account for the nature of the structure by making the model of the errors more complex. For example, methods to estimate a model inadequacy function have been proposed by Kennedy and O'Hagan (2001); to deal with heteroscedasticity by transformation (e.g. Box and Cox, 1964) and to integrate over a distribution of input errors by Kavetski et al. (2002). The aim is to produce an error series for the model error that has a constant variance and (relative) independence in time and space to allow the various parameters and correction terms to be more easily estimated.

In all these approaches, the implicit assumption that the model is correct remains and leaves open the possibility for the (non-physical) structural model of the errors compensating for errors in the model structure and from other sources (Beven, 2004c). Other feasible models that provide acceptable simulations are then commonly neglected. The interest is only in efficiently identifying that true model as some optimum in the space of feasible models. This is understandable in the ideal case because it is known that a "true" model exists. It does not necessarily follow that those other acceptable models are not of interest in more realistic cases where the possibility of model structural error may undermine the assumption that the model is correct (or more generally that the effects of model structural error can be treated simply as a linear contribution to the total model error of Eqn.(1)).

\section{Realistic cases: compensation of uncertainty}

In more realistic cases, it is not possible to assume that the model structure is correct nor is it possible to separate the different sources of model uncertainty. We can assess the series of total model errors in space and time that results from the individual sources of errors in conjunction with the effects of model structural error. In fact, even the true measurement error is independent of model structural error only for the case where predicted variables and observed variables are truly commensurate. If scale, nonlinearity and heterogeneity issues arise in comparing predictions with measurements then the effective measurement error may also interact with model structural error.

There is then significant possibility for calibrated parameter values to compensate for different types of error, perhaps in complex ways. An obvious example is where it is attempted to adjust an input series, such as rainfall inputs to a rainfall-runoff model, in calibration (e.g. Kavetski et al., 2002). At the end of a long dry period it is common for rainfall-runoff models to underpredict stream discharges during the wetting up period. An increase in the rainfalls for the storms during this period will result in smaller model errors (in a nonlinear way), but might also increase soil water storage too much, but this could be compensated by reducing rainfalls in later storms to reduce model errors. The estimated input errors may then be only partially related to real errors in the estimate of rainfall over the catchment area. To make the problem even more intractable, the compensatory effect may be dependent on the particular 
sequence or realisation of the different types of errors, such that assymptotic assumptions are not justified. Certainly, we generally find in optimisation studies that optimal parameter sets are dependent on the period of calibration data used.

There does not appear to be a way around this problem without making some very strong (and generally difficult to justify) assumptions about the nature of the errors. What it does imply, however, is that many different representations (model inputs, model structures, model parameter sets, model errors) might be consistent with the measurements with which the predictions are compared in calibration (allowing for the errors associated with those measurements). Equifinality is endemic to this type of environmental modelling. This would be the case even if we could be sure that we had a set of equations that were a good representation of the processes involved (the "perfect model" of Beven, 2002a, noting that such perfection will never be achievable) but, as is normally the case, only limited information on which to estimate the parameter values of those equations in any particular application.

There are set-theoretic approaches that reject the idea of an optimal model (which might in any case be very dependent on the particular set of measurement and input errors associated with the period of data used) in favour of finding a set of representations (model inputs, model structures, model parameter sets, model errors) that are behavioural in the sense of being acceptably consistent with the (non-error-free) observations (see below). This is the basis of the Generalised Likelihood Uncertainty Estimation (GLUE) methodology of Beven and Binley (1992; Beven and Freer, 2001). There remains the question of how to evaluate whether a model should be considered acceptable or behavioural.

\section{Equifinality and Model Evaluation}

Once the equifinality thesis is given serious consideration for the simulation problem, the question of model evaluation is particularly interesting. It is not just a matter of finding a set of justifiable assumptions about the structure of the total model error (with or without postcalibration validation), or of different errors contributing to the total model error. It is rather a matter of finding a set of models that satisfy some conditions of acceptability or, more importantly, survive tests of rejection as non-behavioural. It is often the case that if model predictions are examined in sufficient detail it will be possible to reject all the available models unless some degree of error is allowed over and above what could be considered to be strict "measurement error". In taking the more realistic view of sources of model error outlined above this is perfectly understandable, even if it creates practical difficulties that we would rather avoid.

However, allowing some degree of error in defining some threshold of acceptability means that there will never be a clear-cut boundary between behavioural and non-behavioural 
models. Monte Carlo experiments show that there is a spectrum of performance across the range of different parameter sets, from the very best found, to ones that are totally unacceptable (see for example, Figure 1). Those that are easily identified as unacceptable can, of course, be rejected straight away. Those that are the very best found would normally be retained as behavioural (or more traditionally as "optimal") but would not necessarily always be adequate in the sense of being entirely consistent with the observations (see Freer et al., 2002). The threshold of acceptability, however, is difficult to define objectively for cases where model structural error is unknown and where the best values of a performance measure found for a particular model tend to vary from application to application. Thus how best to provide a criterion of model acceptability (or rejection) remains an open, but interesting, question.

In applications of the GLUE methodology and other set-theoretic calibration methods, a wide variety of performance measures and rejection criteria have been used in the past. All can be considered as a way of mapping of the hydrological system of interest into a model space (Beven, 2002a,b). Initially, the mapping will be highly uncertain but as more information about the system becomes available, then it should be easier to identify those parts of the model space that give behavioural simulations. The approach is sufficiently general to subsume both traditional optimisation (mapping to a single point in the model space); stochastic identification (mapping to a small region controlled by the identified covariance structure); the equifinality thesis if all behavioural model structures and parameter sets are considered; and hypothesis testing or Bayesian updating in refining the mapping (Beven, 2002a,b; Beven and Young, 2003).

\section{Set Theoretic Methods for model evaluation}

Monte Carlo based set-theoretic methods for model calibration and sensitivity analysis have been used in a variety of disciplines for some 50 years. The first use in geophysics was perhaps that of Press (1968) where a model of the structure of the earth was evaluated in the light of knowledge about 97 eigenperiods, travel times of compressional and shear waves, and the mass and moment of inertia of the earth. Parameters were selected randomly from within specified ranges for 23 different depths which were then interpolated to 88 layers within a spherical earth. Ranges of acceptabilty were set for the predictions to match these

observational data. These were applied successively within a hierarchical sampling scheme for the compressional, stress and density parameters. Five million models were evaluated of which 6 passed all the tests (although of those three were then eliminated as implausible because of having a negligible density gradient in the deep mantle). The "standard model" of the time was also rejected on these tests. Subjective choices were made both of the sampling ranges for the parameters and for the multiple limits of acceptability. Those choices are made explicit, and are therefore open to discussion (indeed, Press discusses an 
additional constraint that might be evoked to refine the results to a single model but notes that "while reasonable, it is not founded in either theory or experiment", p.5233).

Use of this type of Monte Carlo method in hydrology and water quality modelling dates back (at least) to the 1970s (Whitehead and Young, 1979; Hornberger and Spear, 1981; Gardner and O'Neill, 1983; Young, 1983). In many studies the set of feasible models has been defined a priori and the Monte Carlo realisations are then used as a means of propagating prediction uncertainties in a nonlinear modelling context. The more interesting question, however, is to let the available observations condition the behavioural models, without making strong prior assumptions about the parameter distributions or feasible models. This was the essence of the Generalised Sensitivity Analysis of Hornberger and Spear (1981) which was based on assessing all the model realisations into the set of behavioural models and the set of non-behavioural models according to some ranking of model performance. Such studies rapidly found, however, that in many cases there will be no clear demarcation between behavioural and non-behavioural models and, in the case of Hornberger et al. (1985) resort was made to declaring the top $30 \%$ as behavioural in a preliminary sensitivity analysis.

Multiple measures, as in the Press (1968) study, should help in this respect, if a behavioural model is required to satisfy some prior limits of acceptability (see also Hornberger and Spear, 1981). It is possible to define multiple performance measures for a single predicted variable such as discharge (sum of squared errors, sum of absolute errors in peak discharge, sum of squared log errors etc, see for example Parkin et al., 1996) but more information will be added to the conditioning process if a model can be evaluated with respect to distributed observations or multiple chemistry characteristics in water quality.

This does, however, also introduce additional difficulties as soon as it is realised that local observations might require local parameter values to represent adequately the local responses unless generous limits of acceptability are used to allow for the difference in meaning between the prediction at a point by a model using global parameter values (and non-error free input data and model structures) and a local observation. In distributed groundwater modelling, this type of model evaluation suggests that equifinality is endemic to the problem (see Feyen et al., 2001; Binley and Beven, 2003). Similarly, in rainfall-runoff modelling, the use of distributed observational information (disappointingly) does not appear to help much in eliminating the equifinality problem (see Lamb et al., 1998; Blazkova et al., 2002; Blazkova and Beven, 2003; Christiaens and Feyen, 2002).

\section{Extending the concept of the behavioural model}

The concept of such set-theoretic model evaluation is simple. Models that do not fall within the multiple prior limits of acceptability should be rejected. This allows the possibility of many 
feasible models satisfying the limits of acceptability and being accepted as behavioural. It also, however, allows the possibility that none of the models tried will satisfy the limits of acceptability. This was the case for the distributed hydrological model in Parkin et al. (1996), where all parameter sets failed 10 out of 13 limits of acceptability, and for the application of TOPMODEL reported in Freer et al. (2002). It was also the case for a model of the algal dynamics in Lake Veluwe reported in van Straten and Keesman (1991). They had to increase their limits of acceptability by $50 \%$ to obtain any behavioural realisations of the simplest model tried, "to accommodate the apparent structural error" ( $p 175)$.

Thus, any model evaluation of this type needs to take account of the multiple sources of model error more explicitly. As noted above, this is difficult for realistic cases. Simplifying the sources of error to input errors, model structural errors and true measurement errors is not sufficient because of the potential for incommensurability between observed and predicted variables. There is no general theory available for doing this in nonlinear dynamic cases. Most modellers simply assume that they are the same quantity, even where this is clearly not the case. Thus, in assessing model acceptability it is really necessary to decide on an appropriate level of "effective observation error" that takes account of such differences. When defined in this way, the effective observation error need not have zero mean or constant variance, nor need it be Gaussian in nature, particularly where there may be physical constraints on the nature of that error. Once this as been done, then it should be required that any behavioural model should provide all its predictions within the range of this effective observational error. Thus a model will be classified as acceptable if:

$$
Q_{\min }(X, t)<M(\Theta, X, t)<Q_{\max }(X, t) \text { for all } Q(X, t)
$$

Within the range, for all $Q(X, t)$, a positive weight could be assigned to the model predictions, $M(\Theta, X, t)$, according to its level of apparent performance. The simplest possible weighting scheme that need not be symmetric around the observed value, given an observation $Q(X, t)$ and the acceptable range $\left[Q_{\min }(X, t), Q_{\max }(X, t)\right]$ is the triangular relative weighting scheme (Figure 2A)

This is equivalent to a simple fuzzy membership function or relative likelihood measure for the set of all models providing predictions within the acceptable range. A core range of observational ambiguity could be added if required (Figure 2B). Other types of functions could also be used, including the Beta function that is defined by $Q_{\min }, Q_{\max }$ and a shape parameter (Figure $2 \mathrm{C}$ ). These weights for individual data points can be combined in different ways to provide a single weight associated with a particular model. These weights can be used within the GLUE framework in forming prediction limits, reflecting the performance of each behavioural model resulting from this type of evaluation. Models that predict consistently close to the observational data will have a high weight in prediction; those that 
predict outside the acceptable effective observational error will be given zero weight. In forming prediction limits in this way, there is an implicit assumption (as in previous applications of GLUE) that the errors in prediction will be "similar" (in all their complexity) to those in the evaluation period.

Functions with infinite tails, such as the Gaussian distribution, would need to be truncated at the acceptable limits, otherwise the weighting function will also have infinite tails and a poor model would not be rejected, just given a very small likelihood or membership value. This might not be important in statistical inference when seeking an optimal model, but it is important in this context when trying to set limits for acceptable models. For those models that meet the criteria of (3) and are then retained as behavioural, all the methods for combining such measures available from Fuzzy Set Theory are available (e.g. Klir and Folger, 1988; Ross, 1995). Other possibilities of taking account of the local deviations between observed and predicted quantities for the behavioural models, might also be used.

This methodology gives rise to some interesting possibilities. If a model does not provide predictions within the specified range, for any $Q(X, t)$, then it should be rejected as nonbehavioural. Within this framework there is no possibility of a representation of model error being allowed to compensate for poor model performance, even for the "optimal" model. If there is no model that proves to be behavioural then it is an indication that there are conceptual, structural or data errors (though it may still be difficult to decide which are the most important). There is, perhaps, more possibility of learning from the modelling process on occasions when it proves necessary to reject all the models tried.

This implies that consideration also has to be given to input and boundary condition errors, since, as noted before, even the "perfect" model might not provide behavioural predictions if it is driven with poor input data error. Thus, it should be the combination of input/boundary data realisation (within reasonable bounds) and model parameter set that should be evaluated against the observational error. The result will (hopefully) still be a set of behavioural models, each associated with some likelihood weight (Figure 3A). Any compensation effect between an input realisation (and initial and boundary conditions) and model parameter set in achieving success in the calibration period will then be implicitly included in the set of behavioural models.

The explicit acceptance that obtaining a behavioural model depends on a realisation of the input and boundary condition errors does not, however, in itself provide any information on how to construct such realisations. Such errors are likely to be structured and nonstationary in time and space, and dependent on the dynamics of the system. They are most unlikely to be multivariate Gaussian with simple variance and correlation structures. 
There is also the possibility that the behavioural models defined in this way do not provide predictions that span the range of the acceptable error around an observation (Figure 3B). The behavioural models might, for example, provide simulations of an observed variable $Q(X, t)$ that all lie in the range $Q(X, t)$ to $Q_{\max }(X, t)$, or even just a small part of it. They are all still acceptable, but are apparently biased. This provides real information about the performance of the model (and/or other sources of error) that can be investigated and allowed for specifically at that site in prediction (the information on the quantile deviations of the behavioural models, as shown in Figure $3 \mathrm{C}$, can be preserved, for example). Time series of these quantile deviations might provide useful information on how the model is performing across a range of predictions..

This seems to provide a very natural approach to model calibration and evaluation, that avoids making difficult assumptions about the nature of the modelling errors other than specifying the acceptable effective observational error. It also focuses attention on the difference between a model predicted variable (as subject to input and boundary condition uncertainty) and what can actually be observed in the assessment of the effective observational error where this is appropriate; potential compensation between input and structural error; and the possibility of real model failure.

It is always, of course, possible to avoid rejection of all the models tried by extending the range of acceptable error (or adding a compensating statistical error model). This might also depend on the requirements of the modelling application, but the important point is that there would need to be an explicit recognition and argument for doing so. An approach based on rejection rather than optimisation, also tends to focus attention on particular parts of the record that are not well simulated or particular "outlier" errors. In this way we might learn more about model performance (and, hopefully, hypotheses about processes)

\section{Equifinality, confidence limits, tolerance limits and prediction limits}

In statistical inference, a number of different types of uncertainty limits are usually recognised. Hahn and Meeker (1991) for example suggest that confidence limits should contain a specified proportion of some unknown characteristic of a population or process (e.g. a parameter value); tolerance limits should contain some specified proportion of the sampled population or process (e.g. the population of an observed variable); prediction limits should contain a specified proportion of some future observations from a population or process. These simple definitions, underlain by probability theory, do not carry over easily to a situation that recognises multiple behavioural models and the possibility of model structural error.

Whenever predictions of future observations are required, the set of behavioural models can be used to give a prediction range of model variables as conditioned on the process of model 
evaluation. The fuzzy (possibilistic) or probabilistic weights associated with each model can be used to weight the predictions to reflect how well that particular model has performed in the past. The weights then control the form of a cumulative density (possibility) function for any predicted variable over the complete set of behavioural models, from which any desired prediction limits can be obtained. The weights can be updated as new observations are used to refine the model evaluation. This is the essence of the GLUE methodology and of other set theoretic approaches to model prediction (e.g. Beven and Freer, 2001).

Note, however, that while it is necessary to assume that the behavioural models in calibration will also be behavioural in prediction, this procedure only (at best) gives the tolerance limits (in the calibration period) or the prediction limits of the weighted simulations of any variable. These prediction limits will be conditional on the choice of limits of acceptability; the choice of weighting function; the range of models considered; any prior weights used in sampling parameter sets; the treatment of input data error etc. All these components of estimating the uncertainty in the predictions must, at least, be made explicit. However, given the potential for input and model structural errors, they will not guarantee that a specified proportion of observations, either in calibration or future predictions, will lie within the tolerance or prediction limits (the aim, at least, of a statistical approach to uncertainty). Nor is this necessarily an aim in the proposed framework. In fact it would be quite possible for the tolerance limits over all the behavioural models to contain not a single observed value in the calibration period (as in Figure 3B), and yet for all of those models to still remain behavioural in the sense of being within some specified acceptable error limits for all observed quantities. The same could clearly be true in prediction of future observations, even if the assumption that the models remain behavioural in prediction is valid.

Similar considerations apply in respect of the confidence limits for a parameter of the model. Again, it is simple to calculate likelihood weighted marginal distributions of any parameter over all the behavioural models. The marginal distributions can have a useful role in assessing the sensitivity of model outputs to individual parameters (e.g. Hornberger and Spear, 1981; Young, 1983; Beven and Binley, 1992; Beven and Freer, 2001). For each of those models, however, it is the parameter set that results in acceptable behaviour (in conjunction with an input realisation). It is quite possible to envisage a situation in which a parameter set based on the modal value of each of the parameter marginal distributions is not itself behavioural (even if this might be an unlikely scenario). Any confidence limits for individual parameters derived from these marginal distributions therefore cannot have the same meaning as in traditional inference (in the same way that the use of likelihood has been generalised within this framework). Marginal parameter quantiles can, however, be specified explicitly. 
This account of the different uncertainty limits raises a further issue in prediction as to how best to take account of any information on deviations between the behavioural model predictions and observed quantities (as demonstrated in Figure 3C). One approach is the use of probabilistic weights based on a formal likelihood function is then a special case of this procedure for cases where strong (normally Gaussian, with or without bias, heteroscedasticity and autocorrelation) assumptions about the error structure can be justified (see Romanowicz et al., 1994, 1996, who used classical likelihood measures within the GLUE framework). The advantage of doing so is that a formal likelihood function takes account of the residual error in predicting an observed value given the model. The difficulties in doing so are that it adds error model parameters to be identified and that there is no reason to expect that the structural model of the errors should be Gaussian or the same across all the behavioural models (albeit that these are often used as convenient assumptions).

As noted above, an alternative approach based on preserving calibration information on quantile deviations of the behavioural models might be possible. This can be done in a consistent way for any particular observation by transforming the prediction quantiles of the behavioural models to the fuzzy membership function that defines model acceptability (Figure $3 C$ ). In prediction it would then still be necessary to understand how those deviations vary with different conditions (magnitude and ordering of forcing events, different prediction sites, etc) in prediction (see also predicting the impacts of change below). This is the subject of current research, particularly for deviations showing correlation in space and time.

There is a particular difficulty for cases where it is a combination of an input realisation and parameter set that gives a behavioural model. In prediction, it is then easy to use the behavioural parameter sets to provide likelihood weighted predictions as before, but the input data might also be in error in the prediction period. It will not be known a priori which input data realisations will give good predictions with a particular model parameter set, unless analysis of results during the calibration period reveal some strong interaction between the characteristics of an input realisation and a behavioural parameter set. Note, however, that this will be an issue in any prediction problem for which an attempt is made to allow for input data errors, especially if this is done on a forcing event by event basis (e.g. Kavetski et al., 2002).

\section{Equifinality and model validation}

Model validation is a subject fraught with both practical and philosophical undertones (see Stephenson and Freeze, 1974; Konikow and Bredehoft, 1992; Oreskes et al., 1994; Anderson and Bates, 2001; Beven, 1993, 2001b, 2002a,b). The approach outlined in the previous section also provides a natural approach to model validation or confirmation, even when faced with a set of behavioural models. All the time that those models continue to provide 
predictions within the range of the "effective observational error" (allowing for input data errors) they will continue to be validated in the sense of being behavioural. When they do not, they will be rejected as non-behavioural.

There are clearly, however, a number of degrees of freedom in this processes. Stephenson and Freeze (1974) were perhaps the first in hydrology to point out that the dependence of model predictions on input and boundary condition data made strict model validation impossible for models used deterministically, since those data could never be known precisely. The same holds within the methodology proposed here since whether a model is retained as behavioural depends on a realisation of input and boundary condition data. Model evaluation with respect to new data will then be conditional on the input scenarios used to drive the model.

There is also the question of defining the effective observational error. The more error that is considered allowable, the less likely it is that models will be rejected. Clearly, the error limits that are used in any particular study must be chosen on the basis of some reasoning about both the observed and predicted variables, rather than simply making the error limits wide enough to ensure that some models are retained. We do not, after all, learn all that much about the representation of hydrological processes from models that work; we do (or at least should) learn from when we are forced to reject all the available models, even taking account of errors in the process. Strict falsification is not, however, so very useful when in virtually all environmental modelling, there are good reasons to reject models when they are examined in detail (Beven, 2002a; Freer et al., 2002). What we can say is that those models that survive successive evaluations suitable for the application are associated with increasing confirmation (even if not true validation).

\section{Equifinality and model spaces: sampling efficiency issues}

We have noted that acceptance of the equifinality thesis implies that there will be the possibility of different models from different parts of (a generally high dimensional) model space that will provide acceptable simulations, but that the success of a model may depend on the input data sequence used. In one sense, therefore the degrees of freedom in specifying input data sequences will give rise to additional dimensions in the model space.

There is therefore a real practical issue of the equifinality thesis of sampling the model space to find behavioural models (if they exist at all). Success in this endeavour will be dependent on the structure of where behavioural models are found in the space. There is an analogy here with the problem of finding an optimum model on a complex response surface in the model space. The problems of finding a global optimum, rather than local optima has long been recognised and a variety of techniques have been developed to do so successfully. The 
equifinality thesis extends the problem: ideally we require a methodology that both robustly and efficiently identifies those (arbitrarily distributed) regions of the parameter space containing behavioural models, but with the additional dimension that success on finding a behavioural model will depend on a particular realisation of the input variables required to drive the model.

As in any identification problem the search, including modern $\mathrm{MC}^{2}$ methods, can be made much more efficient by making strong assumptions about prior likelihoods for individual parameters and about the shape of the response surface. This seems a little problematic, however, in many environmental modelling problems when it may be very difficult to specify prior distributions for effective values of parameters and their covariation. In the GLUE methodology, the normal (but not necessary) prior assumption has been to specify a feasible range for each parameter, to sample parameter values independently and uniformly within that range in forming parameter sets, and to allow the evaluation of the likelihood measure(s) to condition a posterior distribution of behavioural parameter sets that reflects any interaction between parameters in producing behavioural simulations. This is a simple, minimal assumption approach, but one that will be very inefficient if the distribution of behavioural models within the model space is highly structured. It has the advantage that all the samples from the model space can be considered as independent, although this assumption is not invariant with respect to scale transforms of individual parameter dimensions (e.g. from an arithmetic to a log scale). It is also worth noting that where a model is driven with different realisations of stochastically varying inputs or parameter values, then each point in the model space may be associated with a whole distribution of model outcomes.

\section{Equifinality and model spaces: refining the search}

There may be some possibilities of refining this type of search. The CART approach of Spear et al. (1994) for example, uses an initial set of sample model runs to eliminate regions of the model space where no behavioural models have been found from further sampling. This could, of course, be dangerous where the regions of behavioural models are small with respect to the initial sampling density, though by analogy with some simulating annealing, $\mathrm{MC}^{2}$ and other forms of importance sampling methods, some safeguards against missing some behavioural regions could be ensured by reducing sampling density, rather than totally eliminating sampling, in the apparently non-behaviourly areas.

The only real answer to characterising complex model spaces is, of course, to take more samples. Thus current computational constraints may limit the applicability of the equifinality thesis to a limited range of models. Global circulation models, for example, will certainly be subject to equifinality but are still computationally constrained to the extent that uncertainty in their predictions is essentially limited to a comparison of a small number of deterministic 
simulations (though see www.climateprediction.net). In other cases, it is relatively easy to run billions of sample models within a few days (lorgulescu et al., 2004). The more complex the model, and the longer the run time, then the more constrained will be the number of samples that will be practically feasible. The question is when has a sufficient number of samples been taken to obtain an adequate representation of the different behavioural model functionalities that might be useful in prediction. The answer will vary according to the complexity of the model space. What can be done is to examine the convergence of the outputs from the process (uncertainties in predicted variables or posterior marginal parameter distributions if appropriate) as more samples are added to test whether a sufficient sample of behavioural models has been sampled.

This problem will get less as computer power increases, particularly since it is often easy to implement this type of model space sampling on cheap parallel processor machines. It certainly seems clear that for the foreseeable future, computer power will increase much more quickly than any changes in modelling concepts in hydrology. Thus we should expect that an increasing range of models will be able to be subjected to this type of analysis. Preliminary studies are already being carried out, for example, with distributed hydrological models such as SHE (Christiaens and Feyen, 2002; Vazquez, 2003) and distributed groundwater models (Feyen et al., 2001) albeit with reduced parameter dimensions.

\section{Conclusions}

One reaction to the preceding discussion will almost certainly be that the problems posed by equifinality of models is a transitory problem that will eventually go away as we learn more about hydrological processes and the characteristics of hydrological systems through improved measurement techniques. It is not, therefore, a sufficiently serious problem to warrant throwing away all the useful statistical inference tools developed for model calibration. Within a Bayesian framework, for example, it should be much easier in future to provide good prior distributions of parameter values (and model structures for particular applications) that will provide adequate constraints on the calibration problem and predictive uncertainty.

For forecasting problems involving data assimilation, with the requirement of implementing adaptive algorithms and minimum variance predictions to allow decision making in real time, I would agree. The aim then is to produce optimal forecasts and an estimate of their uncertainty rather than a realistic representation of the system. However, for the simulation problem this is, arguably, a delusion at a time when we cannot demonstrate the validity of the water balance equation for a catchment area by measurement without significant uncertainty (Beven, 2001c). For the foreseeable future it would seem that if equifinality is to be avoided then it will be avoided at the expense of imposing artificial constraints on the modelling problem (such as very strong prior assumptions about model structures, parameter values 
and error structures). It is important to note that the equifinality thesis should be viewed not as simply a problem arising from the difficulty of identifying parameter values but as the identification of multiple functional hypotheses (the behavioural models) about how the system is working (Beven, 2002a,b). Associating likelihood values with the behavioural models, after an evaluation of model errors in calibration, is then an expression of the degree of belief in the feasible hypotheses. Rejection of models as non-behavioural is a refinement of the feasible hypotheses in the model space (which can include multiple model structures as well as parameter sets).

There remains the constraint that all the predictions made are necessarily dependent on how well the model structures considered represent the system responses and the accuracy of the data with which they are driven. Again, the only way of testing whether a model (as functional hypothesis) is adequate is by testing it. It is purely an empirical result that in applications to real systems, with their complexities and data limitations, such testing results in apparent (or real) equifinality.

This analysis of the equifinality thesis has revealed the need for further research in a number of important areas.

- How to define "effective observational error" for cases where the observation and (nonlinear) predictions are not commensurable variables (even if they have the same name).

- How to define limits of acceptability for model predictions, depending on model applications.

- How to separate the effects of model input and structural error and analyse the potential for compensating errors between them.

- How to ensure efficiency in searching model parameter spaces for behavioural models.

- How to allow for the potential deviations between the range of acceptable observational error and behavioural model predictions in calibration when making simulations of new periods, future conditions or "similar" ungauged sites.

- How to deal with the potential for input error in simulation, when it may be particular realisations of inputs that provide behavioural models in calibration.

- How to use model dimensionality reduction to reduce the potential for equifinality, particularly in distributed modelling.

- How to present the resulting uncertainties as conditional probabilities or possibilities to the user of the predictions, together with an explicit comprehensible account of the assumptions used.

These include some difficult research problems, for which it is hard to see a satisfactory resolution in the near future (and it is worth noting that Bruce Beck made a similar list of 
research questions in his review paper of 1987 that shows significant overlap with the questions posed above). Some are common to traditional approaches to model calibration but there is a clear difference in philosophy in the concepts presented here (Baveye, 2004; Beven, 2002a,b; 2004a,c). This manifesto will perhaps not persuade many modellers that there is an alternative (more realistic) way to progress the science of hydrology. The impossibility of separating out the different sources of error in the modelling process allows the difficulties of assessing model structural error to be avoided, and traditional methods of inference to remain attractive. However, this seems naïve. We need better methods to address the model structural error problem, or methods that reflect the ultimate impossibility of unambiguously disaggregating different sources of error. A perspective from an acceptance of the equifinality thesis is, at least, a start in a promising direction.

\section{Acknowledgements}

The work on which this paper is based is supported by NERC Long Term Grant NER/L/S/2001/00658. I would like to thank George Hornberger, Peter Young and Lenny Smith for many fruitful discussions on this topic over a long period, together with all the colleagues and graduate students who have contributed to the practical applications of the equifinality concept, especially Andy Binley, Sarka Blazkova, Rich Brazier, David Cameron, Stewart Franks, Jim Freer, Rob Lamb, Trevor Page, Pep Piñol, Renata Romanowicz, Karsten Schulz, Paul Smith, Jonathan Tawn and Susan Zak. That certainly does not mean that any of them will necessarily agree with the inferences that I have drawn here.

\section{References}

Anderson, M G and Bates, P D (Eds.), Model Validation: Perspectives in Hydrological Science, Wiley: Chichester, 2001.

Bashford, K., Beven, K. J. and Young, P C, Model structures, observational data and robust, scale dependent parameterisations: explorations using a virtual hydrological reality, Hydrol. Process., 16(2), 293-312, 2002.

Baveye, $P$, Emergence of a new kind of relativism in environmental modelling: a commentary, Proc. Roy. Soc. Lond., A460, 2141-2146, 2004.

Beck, M B, Water quality modelling: a review of the analysis of uncertainty, Water Resources Research, 23(8), 1393-1442, 1987.

Beck, M B and Halfon, E, Uncertainty, identifiability and the propagation of prediction errors: a case study of Lake Ontario, J. Forecasting, 10, 135-162, 1991.

Bernardo, J M and Smith, A F M, Bayesian Theory, Wiley, Chichester, 1994.

Beven, K.J., Prophecy, reality and uncertainty in distributed hydrological modelling, $A d v$. Water Resourc., 16, 41-51, 1993. 
Beven, K.J., Equifinality and Uncertainty in Geomorphological Modelling, in B L Rhoads and C E Thorn (Eds.), The Scientific Nature of Geomorphology, Wiley: Chichester, 289-313, 1996.

Beven, K J, TOPMODEL: a critique, Hydrol. Process., 11(9), 1069-1086, 1997.

Beven, $\mathrm{K} \mathrm{J}$, Uniqueness of place and process representations in hydrological modelling, Hydrology and Earth System Sciences, 4(2), 203-213, 2000.

Beven, K J. Rainfall-runoff modelling: the primer, Wiley, Chichester, 2001a.

Beven, $\mathrm{K}$ J. How far can we go in distributed hydrological modelling?, Hydrology and Earth System Sciences, 5(1), 1-12, , 2001b.

Beven, K J, On hypothesis testing in hydrology, Hydrological Processes (HPToday), 15, 1655-1657, 2001c.

Beven, K J. Towards a coherent philosophy for environmental modelling, Proc. Roy. Soc. Lond.,A460, 458, 2465-2484, 2002a.

Beven, K. J., Towards an alternative blueprint for a physically-based digitally simulated hydrologic response modelling system, Hydrol. Process., 16, 2002b.

Beven, K. J., Response to "Emergence of a new kind of relativism in environmental modelling: a commentary", Proc. Roy. Soc. Lond., A460, 2147-2151, 2004a.

Beven, K. J., Does an interagency meeting in Washington imply uncertainty?, Hydrological Processes, 18, 1747-1750, 2004b

Beven, K. J., On the concept of model structural error, Proceedings of the International Workshop on Uncertainty and Precaution in Environmental Modelling, Denmark, 2004c.

Beven, $\mathrm{K} \mathrm{J}$ and Binley, A M, The future of distributed models: model calibration and uncertainty prediction, Hydrological Processes, 6, 279-298, 1992.

Beven, $\mathrm{K} \mathrm{J}$ and Freer, J, Equifinality, data assimilation, and uncertainty estimation in mechanistic modelling of complex environmental systems, J. Hydrology, 249, 11-29, 2001a.

Beven, K J and Freer, J, A Dynamic TOPMODEL, Hydrol. Process.,15(10), 1993-2011, 2001b.

Beven, K J, Freer J, Hankin, B and Schulz, K. The use of generalised likelihood measures for uncertainty estimation in high order models of environmental systems. in Nonlinear and Nonstationary Signal Processing, W J Fitzgerald, R L Smith, A T Walden and P C Young (Eds). CUP, 115-151, 2000.

Beven, $\mathrm{K} \mathrm{J}$ and Freer, J. Equifinality, data assimilation, and uncertainty estimation in mechanistic modelling of complex environmental systems, J. Hydrology, 249, 11-29, 2001.

Binley, A and Beven, $\mathrm{K} \mathrm{J}$, Vadose zone model uncertainty as conditioned on geophysical data, Ground Water, 41(2), 119-127, 2003.

Blazkova, S., Beven, K. J., and Kulasova, A., On constraining TOPMODEL hydrograph simulations using partial saturated area information, Hydrol. Process., 16(2), 441-458, 2002a. 
Blazkova, S and Beven, K J, Flood Frequency Estimation by Continuous Simulation for a Catchment treated as Ungauged (with Uncertainty), Water Resources Research, 38(8), doi: 10.1029/2001/WR000500, 2002b.

Box, G E P and Cox, D R, An analysis of transformations (with discussion), J. Roy. Stat. Soc., B26, 211-252, 1964.

Brazier, R. E., Beven, K. J., Freer, J. and Rowan, J. S., Equifinality and uncertainty in physically-based soil erosion models: application of the GLUE methodology to WEPP, the Water Erosion Prediction Project - for sites in the UK and USA, Earth Surf. Process. Landf., 25, 825-845, 2000.

Cameron, D., Beven, K. and Naden, P., Flood frequency estimation under climate change (with uncertainty). Hydrology and Earth System Sciences, 4(3), 393-405, 2000

Christiaens K, Feyen J, Constraining soil hydraulic parameter and output uncertainty of the distributed hydrological MIKE SHE model using the GLUE framework, Hydrol. Process., 16 (2): 373-391, 2002

Clarke, R T, Statistical Modelling in Hydrology, Wiley: Chichester, 1994

Cushman, J H, On measurement, scale and scaling, Water Resources Research, 22, 129$134,1986$.

Draper, D, Assessment and propagation of model uncertainty, J. Roy. Stat. Soc., B37, 4598,1995

Feyen, L, Beven, K J, De Smedt, F. and Freer, J, Stochastic capture zones delineated within the Generalised Likelihood Uncertainty Estimation methodology: conditioning on head observations, Water Resourc. Res., 37(3), 625-638, 2001.

Freer, J. E., K. J. Beven, and N. E. Peters. Multivariate seasonal period model rejection within the generalised likelihood uncertainty estimation procedure. in Calibration of Watershed Models, edited by Q. Duan, H. Gupta, S. Sorooshian, A. N. Rousseau, and R. Turcotte, AGU Books, Washington, 69-87, 2002.

Gardner, R H and O'Neill, R V, Parameter uncertainty and model predictions: a review of Monte Carlo results. In M B Beck and $G$ van Straten (Eds.) Uncertainty and Forecasting of Water Quality, Springer-Verlag: Berlin, 245-257, 1983

Gupta, H V, Sorooshian, S and Yapo, P O. Towards improved calibration of hydrologic models: multiple and incommensurable measures of information, Water Resourc. Res., 34, 751-763, 1998.

Hahn, G J and Meeker, W Q, Statistical Intervals, Wiley: New York, 1991.

Hall, J W, 2003, Handling uncertainty in the hydroinformatic process, J. Hydroinformatics, 5.4, 215-232.

Hornberger, G M and Spear, R C, An approach to the preliminary analysis of environmental systems, J. Environmental Management, 12, 7-18, 1981.

Howson, C, and Urbach, P. Scientific Reasoning: The Bayesian Approach, $2^{\text {nd }}$ Edn., Open Court, Chicago, IL, 470pp, 1993 
Ibbitt, R P and O'Donnell, T, Fitting methods for conceptual catchment models, J. Hydraul. Div. ASCE, 97, 1331-1342, 1971.

Iorgulescu, I, Beven, K J and Musy, A, 2004, Data-based modelling of runoff and chemical tracer concentrations in the Haute-Menthue (Switzerland) research catchment, Hydrological Processes, in press.

Jakeman, A J., Littlewood, I G and Whitehead, P G. Computation of the instantaneous unit hydrograph and identifiable component flows with application to two small upland catchments, Journal of Hydrology, 117, 275-300, 1990.

Kavetski, D, Franks, S W and Kuczera, G, Confronting input uncertainty in environmental modelling, in Calibration of Watershed Models, edited by Q. Duan, H. Gupta, S. Sorooshian, A. N. Rousseau, and R. Turcotte, AGU Books, Washington, 49-68, 2002.

Kennedy, M C and O'Hagen, A, Bayesian calibration of mathematical models, J. Roy. Statist. Soc., D63 (3), 425-450, 2001.

Kitagawa, G. Monte Carlo filter and smoother for non-Gaussian nonlinear state space models. Journal of Computational and Graphical Statistics, 5, 1-25, 1996.

Klir, G and Folger, T, Fuzzy sets, uncertainty and information, Prentice Hall: Englewood Cliffs, NJ, 1988.

Konikow, L F and Bredehoeft, J D, Groundwater models cannot be validated?, Adv. Water Resourc., 15, 75-83, 1992.

Krzysztofowicz, R, Bayesian system for probabilistic river stage forecasting, J. Hydrology, 268, 16-40, 2002.

Kuczera, G and Parent, E, Monte Carlo assessment of parameter uncertainty in conceptual catchment models: the Metropolis algorithm, J. Hydrology, 211, 69-85, 1998.

Lamb, R., Beven, K.J. and Myrabø, S., Use of spatially distributed water table observations to constrain uncertainty in a rainfall-runoff model., Advances in Water Resources, 22(4), 305-317, 1998.

Martínez-Vilalta, J, Piñol, J and Beven, K J, A hydraulic model to predict drought-induced mortality in woody plants: an application to climate change in the Mediterranean, Ecological Modelling, 155, 127-147, 2002.

Mwakalila, S, P. Campling, J. Feyen, G. Wyseure and K. J Beven, Application of a databased mechanistic modelling (DBM) approach for predicting runoff generation in semiarid regions, Hydrological Processes, 15, 2281-2295, 2001.

O'Neill, R V, Error analysis of ecological models in Nelson, D J (ed.), Radionuclides in Ecosystems, NTIS: Springfield, 898-908, 1973.

Oreskes, N, Schrader-Frechette, K and Belitz, K, Verification, validation and confirmation of numerical models in the earth scicens, Science, 263, 641-646, 1994.

Page, T, Beven, $\mathrm{K}$ J, Freer, J and Jenkins, A, Investigating the uncertainty in predicting responses to atmospheric deposition using the Model of Acidification of Groundwater in Catchments (MAGIC) within a Generalised Likelihood Uncertainty Estimation (GLUE) framework, Water, Air, Soil Pollution, 142, 71-94, 2003. 
Romanowicz, R., K.J. Beven and J. Tawn, Evaluation of predictive uncertainty in non-linear hydrological models using a Bayesian approach, in V. Barnett and K.F. Turkman (Eds.) Statistics for the Environment II . Water Related Issues, Wiley, 297-317, 1998.

Romanowicz, R., K.J. Beven and J. Tawn, Bayesian calibration of flood inundation models, in M.G. Anderson, D.E.Walling and P. D. Bates, (Eds.) Floodplain Processes, 333-360, 1996.

Romanowicz, R and Beven, $\mathrm{K} \mathrm{J}$, Dynamic real-time prediction of flood inundation probabilities, Hydrol. Sci. J., 43(2), 181-196, 1998.

Ross, T J, Fuzzy Logic with Engineering Applications, McGraw-Hill: New York, 1995

Schulz, K, Beven, K and Huwe, B, Equifinality and the problem of robust calibration in nitrogen budget simulations, Soil Sci. Soc. Amer. J., 63(6), 1934-1941, 1999.

Schulz, K., and Beven, K. Towards simplified robust model structures in land surface atmosphere flux predictions, Hydrol. Process. 17, 2259-2277, 2003.

Stephenson, G R and Freeze, R A, Mathematical simulation of subsurface flow contributions to snowmelt runoff, Reynolds Creek, Idaho, Water Resources Research, 10(2), 284298, 1974.

Thiemann, M, Trosset, M, Gupta, H and Sorooshian, S, Bayesian recursive parameter estimation for hydrologic models, Water Resourc. Res., 37(10), 2521-2535, 2001.

Van Straten, G and Keesman, $\mathrm{K} \mathrm{J}$, Uncertainty propagation and speculation in projective forecasts of environmental change, J. Forecasting, 1991.

Vazquez, R, Assessment of the performance of physically based distributed codes simulating medium size hydrological systems, PhD Thesis, Katolieke Universiteit Leuven, Belgium (ISBN 90-5682-416-3), 335pp, 2003.

von Bertalanffy, L., General Systems Theory, George Brazillier: New York, 1968.

Vrugt JA, Bouten W, Gupta, H V and Sorooshian S, Toward improved identifiability of hydrologic model parameters: the information content of experimental data, water Resour. Res., 38(12), doi:10.1029/2001WR001118, 2002

Vrugt, J A, Gupta, H V, Bouten, W and Sorooshian, S, 2003, A shuffled complex evolution Metropolis algorithm for optimization and uncertainty assessment of hydrologic model parameters, Water Resour. Res., 39(8), doi:10.1029/2002WR001642, 2003.

Whitehead, P G and Young, P C, Water quality in river systems: Monte-Carlo analysis, Water Resources Research, 15, 451-459, 1979.

Yapo, P O, Gupta, H and Sorooshian, S. Multi-objective global optimisation for hydrologic models, J. Hydrol., 204, 83-97, 1998.

Young, P C, The validity and credibility of models for badly-defined systems. In M B Beck and $G$ van Straten (Eds.) Uncertainty and Forecasting of Water Quality, SpringerVerlag: Berlin, 69-98, 1983

Young, P C. Recursive Estimation and Time Series Analysis, Springer-Verlag, Berlin, 1984. 
Young, P C. Recursive estimation, forecasting and adaptive control. In C.T. Leondes (Ed.), Control and Dynamic Systems: Advances in Theory and Applications, Vol. 30, Academic Press: San Diego, 119-166, 1990.

Young, P C. Data-based mechanistic modelling of environmental, ecological, economic and engineering systems, Environmental Modelling and Software, 13, 105-122, 1998.

Young, P C. Data-based mechanistic modelling and validation of rainfall-flow processes, in Anderson, M G and Bates, P D (Eds), Model Validation: Perspectives in Hydrological Science, Wiley, Chichester, 117-161, 2001.

Young, P. C., Advances in Real Time Forecasting, Phil. Trans. Roy. Soc. Lond., A360, 14301450, 2002.

Young, P C., Top-down and data-based mechanistic modelling of rainfall-flow dynamics at the catchment scale, Hydrological Processes, 17, 2195-2217, 2003.

Young, P C, Chotai, A and Beven, K J, 2004, Data-Based Mechanistic Modelling and the Simplification of Environmental Systems, in J. Wainwright and M. Mulligan (Eds.), Environmental Modelling: Finding Simplicity in Complexity, Wiley, Chichester, 371-388.

Young, P C and Parkinson, S, Simplicity out of complexity, in M B Beck (Ed.), Environmental Foresight and Models: A Manifesto, 251-301, 2002.

Zak, $\mathrm{S}$ and Beven, $\mathrm{K} \mathrm{J}$, Equifinality, sensitivity and uncertainty in the estimation of critical loads, Science of the Total Environment, 236, 191-214, 1999.

Zin, I, Incertitudes et ambigüité dans la modélisation hydrologique, Thèse de Doctorat, Institut National Polytechnique de Grenoble, Grenoble, France, 2002. 


\section{Figure Headings}

Figure 1. Dotty plots (projections of points on a likelihood surface onto a single parameter axis) resulting from Monte Carlo realisations of parameter sets for the MAGIC Long Term Soil Acidification and Water Quality Model (after Page et al., 2003). Only 6 out of 12 parameters varied shown. Model evaluation based on joint fuzzy membership function as to whether modelled concentrations fall within acceptable limits for several specific points in time.

Figure 2. Defining acceptable error around an observed value (vertical line), with the observed value, $Q$, not central to the acceptable range, $Q_{\min }$ to $Q_{\max }$. $A$. Triangular, with peak at observation. B. Trapezoidal, with inner core range of observational ambiguity. C. Beta distribution with defined range limits.

Figure 3A. Histogram of simulated values over the set of behavioural models during calibration that include the observed value (indicated by vertical line).

Figure 3B Histogram of simulated values over the set of behavioural models during calibration that do not include the observed value (indicated by vertical line).

Figure 3C Cumulative distributions for the set of behavioural simulation predicted values relative to cumulative distribution of likelihood measure of a single observation, illustrating the concept of quantile deviations (solid arrows: $50 \%$ quantile deviation; spots: $25 \%$ and $75 \%$ quantile deviations; open arrows, $5 \%$ deviation). 

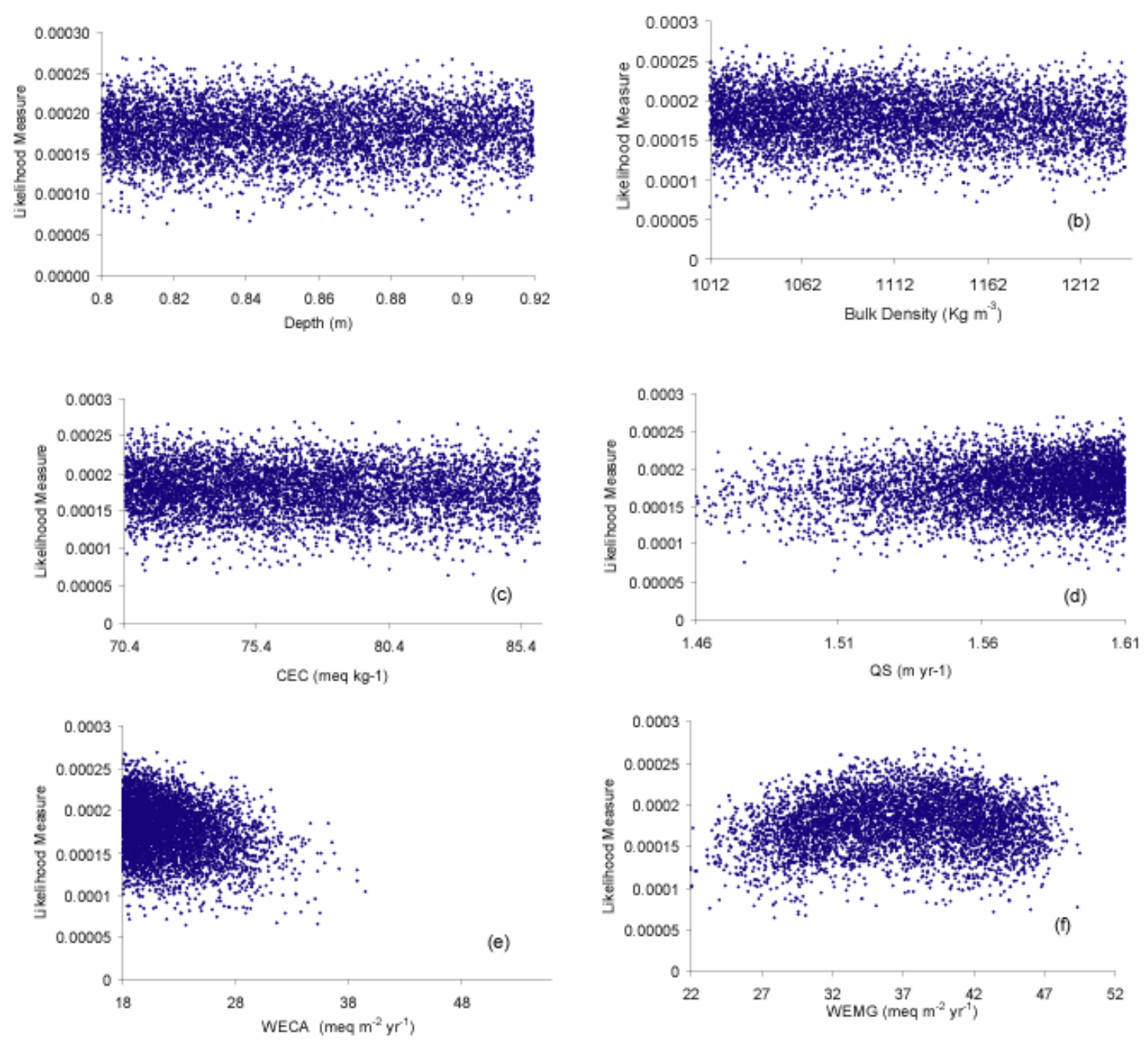

Fig. 1 

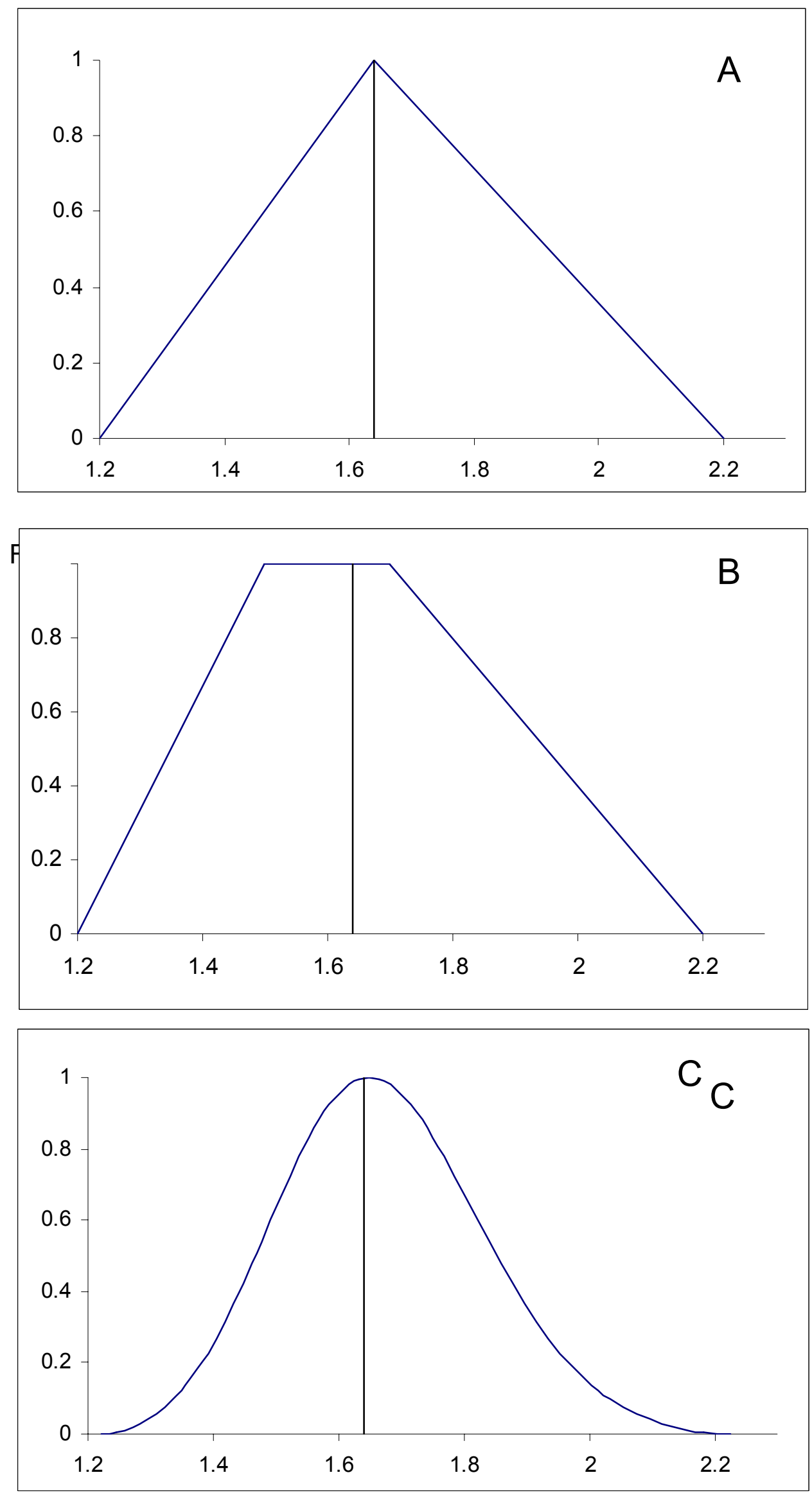

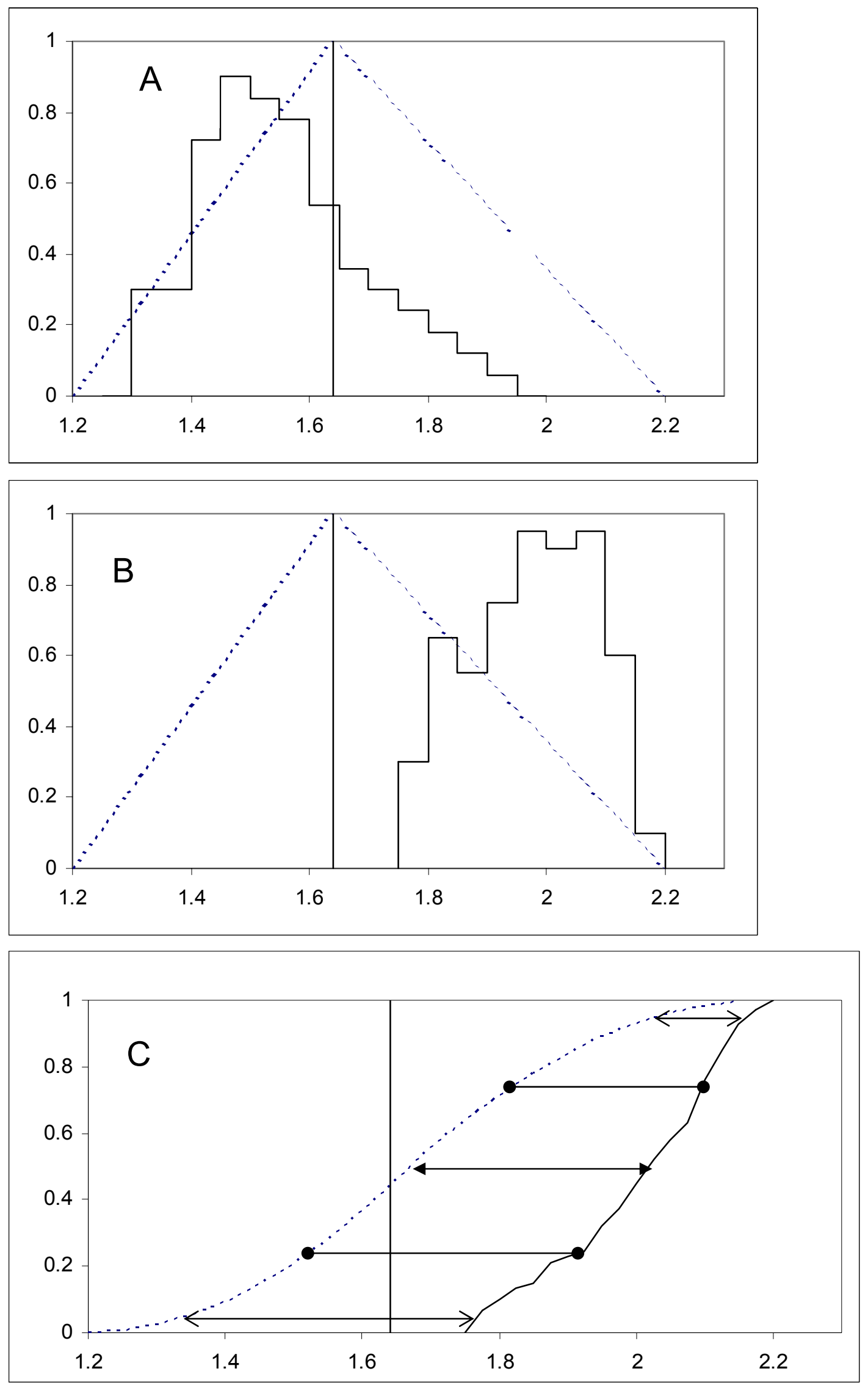

Fig. 3 